\title{
Pulse-Echo Ultrasonic Testing of Adhesively Bonded Joints in Glass Façades
}

\author{
Bor Mojškerc - Tomaž Kek - Janez Grum* \\ University of Ljubljana, Faculty of Mechanical Engineering, Slovenia
}

This article presents a pulse-echo ultrasonic method of evaluation of adhesively bonded joints in glass façades by means of the amplitudes of reflected ultrasonic signals. Glass façade specimens consist of three basic components: hardened glass, polysulfide or silicone adhesive and a polymer profile. Each component has its own specific acoustic impedance, which relates to a difference in the ultrasound reflection and transmission coefficients. Several glass façade specimens containing defects like air pockets, grease and duct tape are analysed. Measurements are carried out using a normal incidence piezoelectric transducer. Experiments are performed on several adhesively bonded joints in order to detect the defects in the glass-adhesive and adhesive-polymer profile interfaces. Most of the defects, except smaller areas of grease, can be successfully detected. The experimental results suggest that pulse-echo ultrasonic testing is a reasonable way of evaluating of adhesively bonded joints in glass façades.

Keywords: ultrasonic testing, pulse-echo method, adhesively bonded joints, glass façades

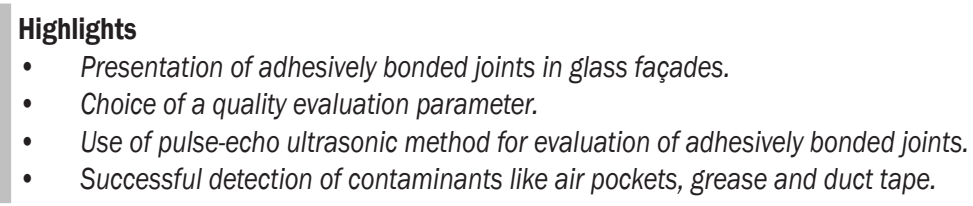

\section{O INTRODUCTION}

The development of civil engineering and materials science have paved the way for the use of glass façades in an array of different structures. Glass façades are commonly made with hardened glass and polymer profiles, joined together by polysulfide or silicone adhesive. These adhesively bonded joints are required to perform according to predetermined specifications. Joints with low mechanical strength present a flaw in the integrity of the structure and pose a danger in the case of joint failure. In order to prevent low quality bonding, joints have to be evaluated during production and lifecycle. Therefore, a reasonable quality control method is needed.

Adhesively bonded joints can be evaluated using different destructive and non-destructive methods: tensile failure tests, mechanical impedance analysis, as well as thermography and ultrasonic methods. A relationship between a lower bond strength threshold due to contaminants and non-destructive test results can be established [1]. Ultrasonic methods offer an opportunity to evaluate adhesively bonded joints without their mechanical failure. Joint strength cannot be directly measured, but can be indirectly evaluated using the chosen ultrasonic parameters in relation to destructive test results.

The through transmission ultrasonic method of ultrasonic inspection can be used to monitor crosslinking of the adhesive during the curing process [2]. Changes in the transmission spectrum are related to adhesive properties and optimal curing time can be determined. The through transmission method can also be used to evaluate fatigue damage, which generates ultrasonic harmonics in the adhesive layer [3]. An increase in fatigue cycles is related to an increase in the acoustic nonlinearity parameter and early damage can be detected. The high frequency through transmission technique can also be used to evaluate nonlinear behaviour of kissing bonds in adhesively bonded joints [4]. Kissing bonds are a special case, where the adherend and adhesive are in intimate contact, but joint strength is minimal or non-existent. The authors suggest that the measured nonlinearity is highly dependent on the ratio between adhesive layer thickness and ultrasonic wavelength.

The pulse-echo ultrasonic method can be used to detect void disbonds at the front and rear interfaces of adhesively bonded joints in automotive assemblies [5]. Absence of disbonding at the front metal-adhesive interface can be indicated by large deviations in the ultrasonic waveform. At the rear interface, the phenomena of phase inversion can be used as an indication of bond quality. Another application of the pulse-echo method is evaluation of adhesion quality using amplitude ratios of multiple successive reflections [6]. 
Oblique incidence pitch-catch ultrasonic inspection can be used to evaluate the degradation of adhesively bonded joints of carbon fiber reinforced plastics (CFRP) [7]. Degraded joints exhibit decreased stiffness, a higher reflected signal amplitude and a shift in the frequency minimum towards lower frequencies. A correlation can be established with adhesive bond strength and an assessment can be made regarding the bond quality using this method.

Another example of ultrasonic testing of adhesively bonded joints is the use of shearhorizontally ( $\mathrm{SH}$ ) polarized guided waves [8]. These can be used to quantify the adhesive shear modulus and interfacial shear stiffness as indicators of adhesive bond quality.

The aim of this article is to develop a way of evaluating of adhesively bonded joints in glass façades. For this purpose, pulse-echo ultrasonic tests are performed on various adhesive bond specimens with different flaws and contaminants like air pockets, grease and duct tape. The amplitudes of the reflected ultrasonic signals are used as a quality evaluation parameter and the applicability of the presented method is determined.

\section{METHODS AND EXPERIMENTAL}

The standard pulse-echo ultrasonic method is based on the emission and reception of sound by an ultrasonic transducer, which is commonly piezoelectric. Generated ultrasonic sound waves travel through a chosen substance and are reflected back at boundary interfaces due to a difference in specific acoustic impedance, which is given by Eq. (1):

$$
Z=\rho c,
$$

where $Z$ is the specific acoustic impedance $\left[\mathrm{Ns} / \mathrm{m}^{3}\right]$, $\rho$ density $\left[\mathrm{kg} / \mathrm{m}^{3}\right]$ and $c$ the sound velocity $[\mathrm{m} / \mathrm{s}]$. A greater difference relates to a greater percentage of reflection. This indicates the possibility of differentiation of substances based on their specific acoustic impedance and the reflected signal amplitude. The quality of adhesively bonded joints in glass façades can therefore be evaluated via comparison of test results with a predetermined acceptable reflected signal amplitude.

The coefficients of reflection and the transmission of sound can be determined via the specific acoustic impedance of substances sharing an interface. They are given by Eqs. (2) and (3):

$$
R=\frac{Z_{2}-Z_{1}}{Z_{2}+Z_{1}}
$$

$$
T=\frac{2 Z_{2}}{Z_{2}+Z_{1}},
$$

where $R$ is the coefficient of reflection [\%], $T$ the coefficient of transmission [\%] and $Z_{1}, Z_{2}$ the specific acoustic impedance of the first and second given substance, respectively $\left[\mathrm{Ns} / \mathrm{m}^{3}\right]$.

\subsection{Equipment and Parameters}

Experiments were performed using a Eurosonic UTC-110 ultrasonic device with attached software. A normal incidence Eurosonic T0506 piezoelectric transducer with a $6 \mathrm{~mm}$ diameter and a $4.75 \mathrm{MHz}$ pulse frequency was used. A summary of the testing parameters is provided in Table 1.

Table 1. Testing parameters

\begin{tabular}{lc}
\hline Parameter & Value \\
\hline Pulse frequency & $4.75 \mathrm{MHz}$ \\
\hline Voltage & $50.15 \mathrm{~V}$ \\
\hline Pulse width & $210 \mathrm{~ns}$ \\
\hline Frequency of pulses & $1 \mathrm{kHz}$ \\
\hline Sampling rate & $100 \mathrm{MHz}$ \\
\hline Amplification & $13.6 \mathrm{~dB}$ to $16 \mathrm{~dB}$ \\
\hline
\end{tabular}

\subsection{Specimens}

A model of the glass façade specimen is presented in Fig. 1. Dimensions of the specimen are $300 \mathrm{~mm} \times 200$ $\mathrm{mm} \times 109 \mathrm{~mm}$. Width of the adhesively bonded joint is $30 \mathrm{~mm}$. A schematic view of the joint is presented in Fig 2. The joint has three basic components with various thicknesses: hardened glass with a thickness of $8 \mathrm{~mm}$, polysulfide or silicone adhesive with a thickness of $3.2 \mathrm{~mm}$ and a polyamide PA6.6 polymer profile with a thickness of $2 \mathrm{~mm}$.

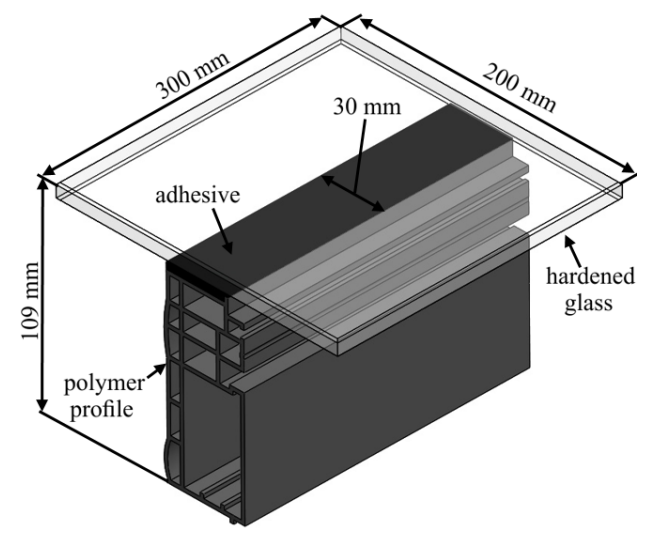

Fig. 1. Glass façade specimen 


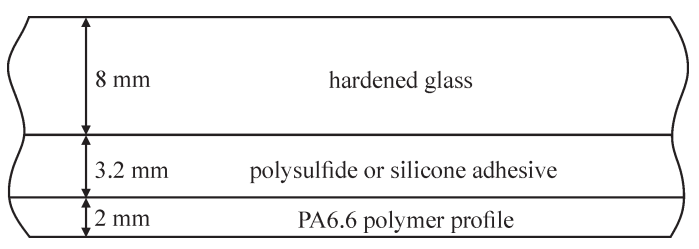

Fig. 2. Schematic view of the adhesively bonded joint

\section{RESULTS AND DISCUSSION}

In this section, several tests of adhesively bonded joints in glass façades are presented. Flaws in glass façades can occur on both interfaces between the glass-adhesive and adhesive-polymer profile. In order to properly evaluate the joint, testing of both interfaces is required. Experiments were performed separately on each interface. The effect of the coupling quality between the ultrasonic transducer and a given material was taken into account. In the case of the glassadhesive interface, the reflected signal amplitude was amplified for each measurement in order to match the amplitude of the first reflection. This was however not possible in the case of the adhesive-polymer profile interface, as the amplitude of the first reflection was later used as a quality evaluation parameter. Test results on both interfaces are presented in A-scan figures of amplitude over time or length of the joint.

\subsection{Interface Glass-Adhesive}

The specimen for the glass-adhesive interface test was produced using a polysulfide adhesive. The normal incidence ultrasonic transducer was positioned on the upper side of the glass. Several measurements were taken in different areas of the joint with or without adhesive. The reflected signal amplitude for a bond with adhesive present is presented in Fig. 3. The reflected signal amplitude for an area with no adhesive is presented in Fig. 4. The reflected echo signal decay is greater in cases where adhesive is present, due to a greater part of the sound pressure passing through the adhesive than through air, as the specific acoustic impedance of air is lower than that of the adhesive. The coefficient of reflection is therefore lower in the case where adhesive is present. A comparison of echo decay for both cases is presented in Fig. 5. The amplitude of the main ultrasonic echoes, marked as $A_{i}$, was determined using the absolute value of the main ultrasonic echoes. The index $i$ indicates the number of the main echo.

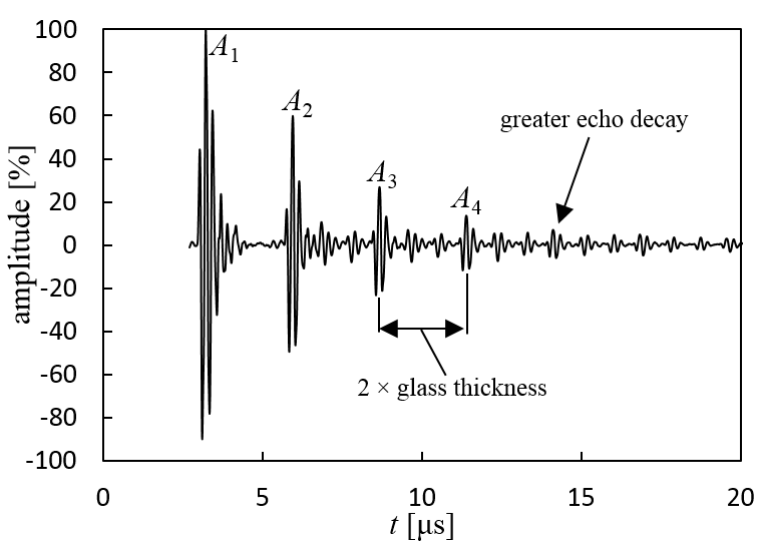

Fig. 3. Reflected signal amplitude at the glass-adhesive interface, with adhesive present

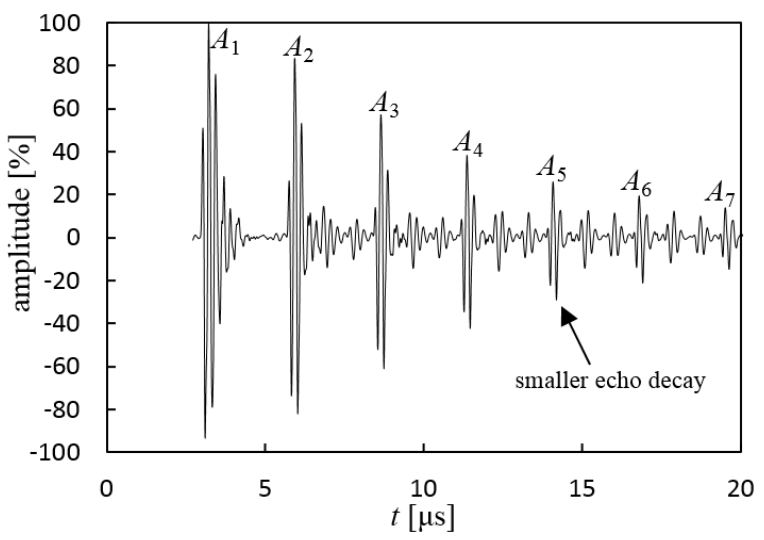

Fig. 4. Reflected signal amplitude at the glass-adhesive interface, with adhesive absent

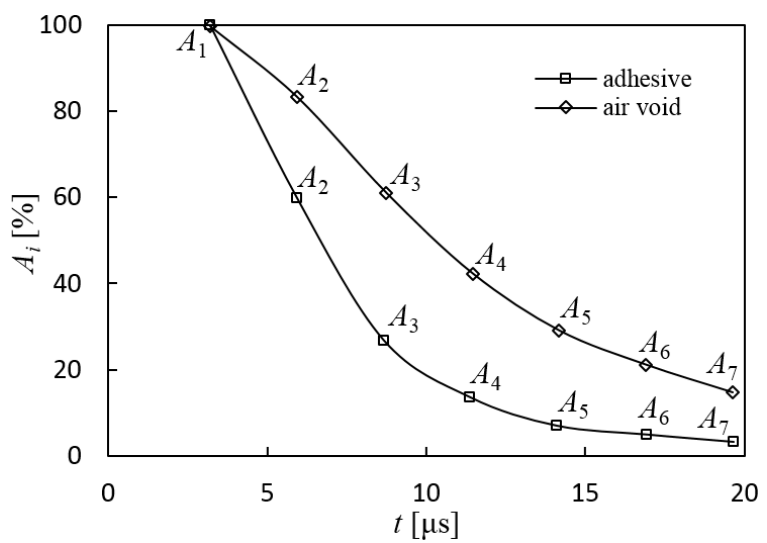

Fig. 5. Comparison of echo amplitude decay at the glass-adhesive interface

\subsection{Interface Adhesive-Polymer Profile}

In the test of the adhesive-polymer profile interface, the specimen was produced using a silicone adhesive. In order to position the ultrasonic transducer on the 
lower side of the polymer profile, the profile had to be partially cut. As before, measurements were taken in separate areas with adhesive either present or absent. The echo amplitude in the adhesive-polymer profile interface with adhesive present is presented in Fig. 6. The echo amplitude in adhesive-polymer profile interface with adhesive absent is presented in Fig. 7. The acoustic impedance match of the adhesivepolymer profile interface is greater than that of the glass-adhesive interface, therefore the echo amplitudes are smaller and only one main echo $A_{1}$ can be clearly seen in the case where adhesive is present. The echo $A_{x}$ is reflected from the glass-adhesive interface and is not visible in the case where adhesive is absent, as the ultrasonic waves reflect at the interface where adhesive is absent and do not reach the glass-adhesive interface.

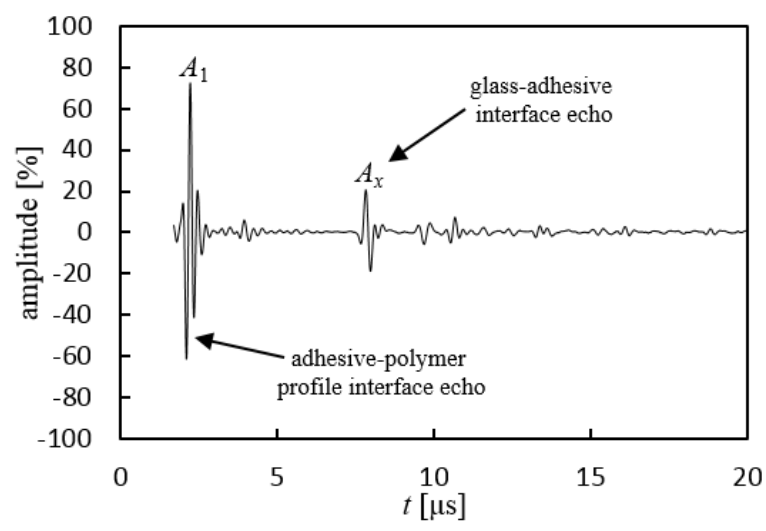

Fig. 6. Reflected signal amplitude at the adhesive-polymer profile interface, with adhesive present

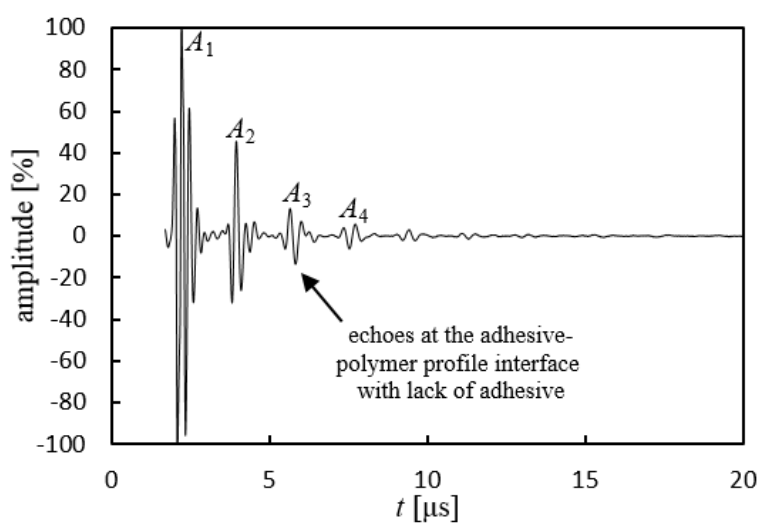

Fig. 7. Reflected signal amplitude at the adhesive-polymer profile interface, with adhesive absent

\subsection{Quality Evaluation Parameter}

In order to evaluate the quality of adhesively bonded joints in glass façades, a parameter from the measured ultrasonic echoes had to be chosen. For the evaluation of the glass-adhesive interface, second, third and fourth reflected echo amplitudes were chosen along with attenuation coefficient $\alpha[\mathrm{dB} / \mathrm{mm}]$, which is given by Eq. (4):

$$
\alpha=\frac{20}{2 s} \log \frac{A_{1}}{A_{2}},
$$

where $s$ is specimen thickness [mm], $A_{1}$ first echo amplitude [\%] and $A_{2}$ second echo amplitude [\%]. A comparison was made for bonds with and without adhesive. Ten measurements were taken. The average values are presented in Table 2. Single factor analysis of variance (ANOVA) was used to identify the greatest difference in the measured mean values, as presented in Tables 3 to 6. A standard ANOVA alpha level of 0.05 was used. The critical $F$ value was 4.41 for all cases, based on the degrees of freedom, $d f$. The term $S S$ abbreviates the sum of squares and $M S$ abbreviates mean squares. The null hypothesis suggests that the mean values of the chosen quality evaluation parameters do not significantly differ in the case of a bond with present or absent adhesive. The null hypothesis was rejected for all cases. The third echo amplitude $A_{3}$ was chosen as a final quality evaluation parameter for the glass-adhesive interface, as it had the greatest mean value difference of reflected signals between bonds. In order to evaluate the adhesivepolymer profile interface, the first echo amplitude $A_{1}$ was chosen, as it was the only clearly present echo in both cases of a bond with or without adhesive.

Table 2. Average values of amplitude and attenuation

\begin{tabular}{lcccc}
\hline Joint type & $A_{2}[\%]$ & $A_{3}[\%]$ & $A_{4}[\%]$ & $\alpha[\mathrm{dB} / \mathrm{mm}]$ \\
\hline Adhesive & 60.46 & 26.42 & 14.13 & 0.28 \\
\hline Air void & 84.31 & 60.83 & 42.02 & 0.09 \\
\hline
\end{tabular}

Table 3. Analysis of variance for echo amplitude $A_{2}$

\begin{tabular}{lcccc}
\hline Variance source & SS [\%2] & $d f$ & $\mathrm{MS} \mathrm{[ \% 2]}$ & $F_{0}$ \\
\hline Between groups & 2843.79 & 1 & 2843.79 & 486.67 \\
\hline Within group & 105.18 & 18 & 5.84 & \\
\hline Total & 2948.97 & 19 & $P_{\text {value }}=1.76 \mathrm{E}-14$ \\
\hline
\end{tabular}

Table 4. Analysis of variance for echo amplitude $A_{3}$

\begin{tabular}{lcccc}
\hline Variance source & SS [\% $\left.{ }^{2}\right]$ & $d f$ & MS [\%2] & $F_{0}$ \\
\hline Between groups & 5920.37 & 1 & 5920.37 & 2199.26 \\
\hline Within group & 48.46 & 18 & 2.69 & \\
\hline Total & 5968.82 & 19 & $P_{\text {value }}=2.85 \mathrm{E}-20$ \\
\hline
\end{tabular}


Table 5. Analysis of variance for echo amplitude $A_{4}$

\begin{tabular}{lcccc}
\hline Variance source & SS [\%2] & $d f$ & MS [\%2] & $F_{0}$ \\
\hline Between group & 3889.43 & 1 & 3889.43 & 1046.09 \\
\hline Within group & 66.93 & 18 & 3.72 & \\
\hline Total & 3956.36 & 19 & $P_{\text {value }}=2.12 \mathrm{E}-17$ \\
\hline
\end{tabular}

Table 6. Analysis of variance for attenuation $\alpha$

\begin{tabular}{lcccc}
\hline Variance source & $\mathrm{SS}\left[\mathrm{dB}^{2} / \mathrm{mm}^{2}\right]$ & $d f$ & $\mathrm{MS}\left[\mathrm{dB}^{2} / \mathrm{mm}^{2}\right]$ & $F_{0}$ \\
\hline Between group & 0.1830 & 1 & 0.1830 & 151.82 \\
\hline Within group & 0.0217 & 18 & 0.0012 & \\
\hline Total & 0.2047 & 19 & $P_{\text {value }}=3.29 \mathrm{E}-10$ \\
\hline
\end{tabular}

The area of quality was determined by the average value of the measured echo amplitude, $A_{1}$ and $A_{3}$, each at its own respective interface. Adhesive bond quality was acceptable if the value of the chosen quality evaluation parameter was inside the area of quality. A sample standard deviation of the echo amplitude was calculated from twenty measurements over an area with adhesive present and no defects. Amplitude limits of the area of quality were determined with Eqs. (5) and (6). Eq. (5) was used to assess the area of quality at the glass-adhesive interface, Eq. (6) was used to assess the area of quality at the adhesivepolymer profile interface:

$$
\begin{gathered}
Q_{g-a}=A_{3 a v g} \pm 2 s_{3}, \\
Q_{a-p p}=A_{1 a v g} \pm 2 s_{1},
\end{gathered}
$$

where $Q_{g-a}$ is the area of quality for the glassadhesive interface [\%], $Q_{a-p p}$ the area of quality for the adhesive-polymer profile interface [\%], $A_{\text {iavg }}$ the average echo amplitude [\%] and $s_{i}$ the sample standard deviation [\%]. Each area of quality is specific to a given specimen and interface. Different types of glass façades cannot use the same amplitude limits. They have to be determined separately for each type, if this method of quality evaluation is to be applied.

\subsection{Testing Joint Quality}

In order to test the applicability of the presented method and the quality evaluation parameters, several adhesively bonded joints were evaluated along the length of the glass façade. A specimen was produced with a purposefully wavy deposit of polysulfide adhesive in order to clearly present the difference between a bond with and without adhesive at the glass-adhesive interface. A top view of the specimen is presented in Fig. 8 together with the test results.
Measurements were taken every $5 \mathrm{~mm}$ in a straight line. The third echo amplitude $A_{3}$ was recorded and plotted against joint length. The quality of the joint was unacceptable if the third echo amplitude left the predetermined area of quality. Air voids were easily detected, as they were outside the area of quality. The amplitude difference between a good bond and a bond with a lack of adhesive was as large as $35 \%$. Small air pockets were also detected and confirmed by visual inspection. The transducer diameter was $6 \mathrm{~mm}$, so only a part of the actual joint width was tested. A matrix array ultrasonic transducer is recommended in order to evaluate the quality of the entire joint.
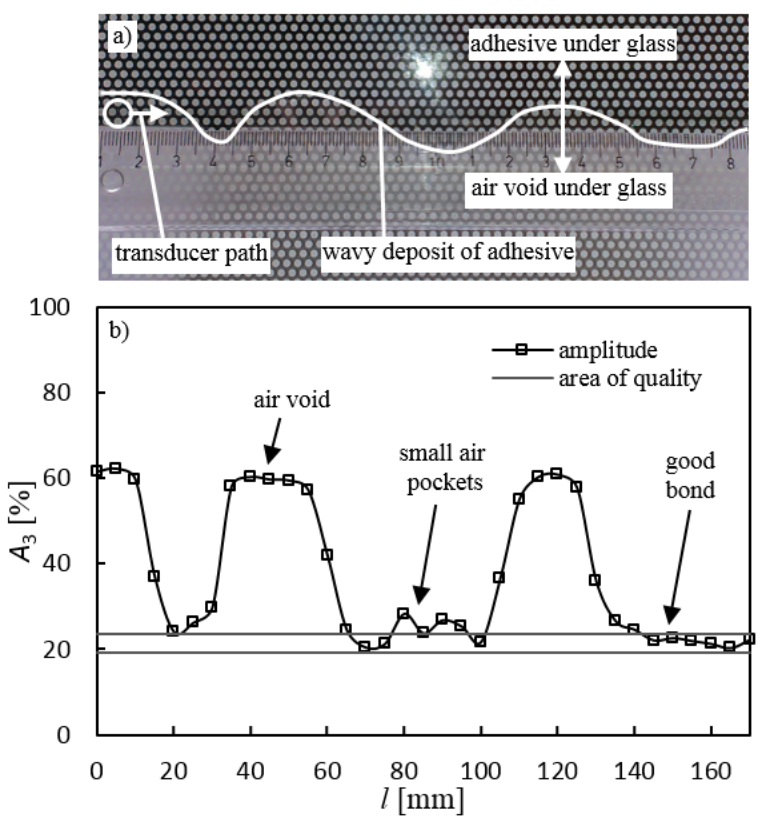

Fig. 8. Test of the glass-adhesive interface: a) top view of the specimen and b) third echo amplitude along the joint length

The next specimen was made with silicone adhesive together with interrupted areas of contaminants on the glass-adhesive interface. Contaminants consisted of two areas of grease and an area of duct tape. The tested area of the specimen is presented in Fig. 9 together with the contaminants and the recorded third echo amplitude $A_{3}$. Measurements were taken every $3 \mathrm{~mm}$ in a straight line. The results show the limits of contaminant detection at the glassadhesive interface. The second area of grease was detected, while the first area of grease remained undetected. The duct tape area was successfully detected, as shown by a more than a $27 \%$ increase in the third echo amplitude. Contaminant detection in the glass-adhesive interface was therefore dependent on 
contaminant type and its arrangement. Very thin areas of grease were difficult to detect.

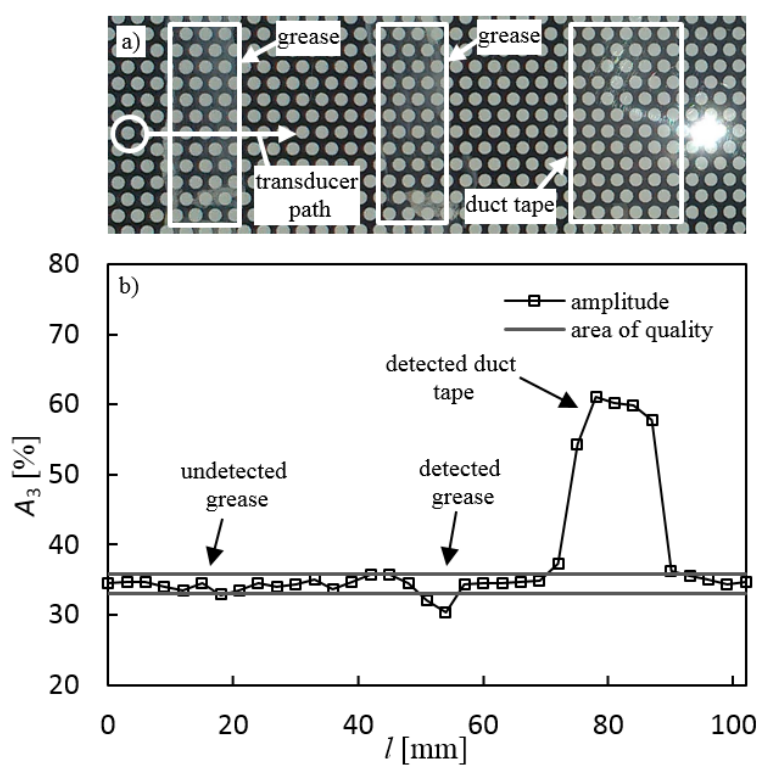

Fig. 9. Test of the glass-adhesive interface with contaminants: a) top view of the specimen and b) third echo amplitude along the joint length
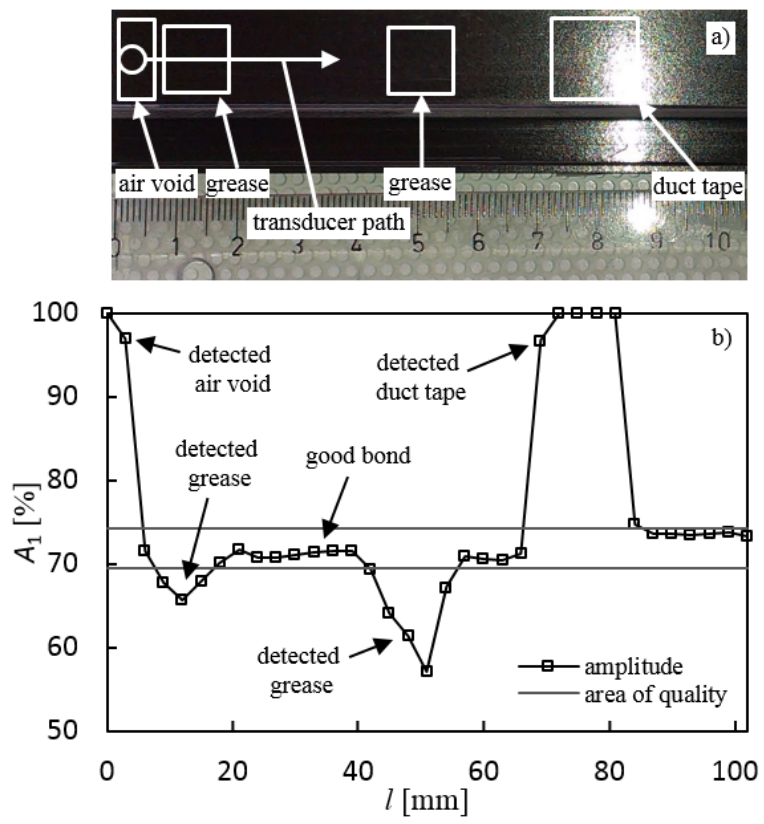

Fig. 10. Test of the adhesive-polymer profile interface with contaminants: a) top view of the specimen and b) first echo amplitude along the joint length

As mentioned before, both glass-adhesive and adhesive-polymer profile interfaces have to be evaluated. The next specimen was made with a deposit of silicone adhesive together with contaminants on the adhesive-polymer profile interface. A top view of the specimen is presented in Fig. 10 together with the contaminants and test results. Measurements were taken every $3 \mathrm{~mm}$ in a straight line. As per the choice of the quality evaluation parameter, the first echo amplitude $A_{1}$ was recorded and plotted against the length of the glass façade. As before, the contaminants consisted of two areas of grease and an area of duct tape. There was also an air void present at the beginning of the joint. The polymer profile is opaque, therefore the contaminants cannot be seen clearly. All contaminants were successfully detected, even the thin areas of grease. Contaminant detection was more successful in the adhesive-polymer profile interface than in the glass-adhesive interface.

\section{CONCLUSIONS}

This paper presented a non-destructive way of evaluating the quality of adhesively bonded joints in glass façades using the pulse-echo ultrasonic method. Adhesive bond quality can be evaluated via the amplitude of reflected echo signals, which differ in relation to the specific acoustic impedance of a given material's interface. Two quality evaluation parameters were chosen. For evaluating the glass-adhesive interface, the third echo amplitude $A_{3}$ was chosen on the basis of a single factor analysis of variance. For evaluating the adhesive-polymer profile interface, the first echo amplitude $A_{1}$ was chosen, as it was the only echo present in both cases of a bond with and without adhesive. Acceptable areas of quality bonding were determined for each specific type of adhesive and interface separately, using the same applied theory. This method of quality evaluation can be used to detect air voids and contaminants like grease and duct tape on both bond interfaces, although very thin areas of grease are hard to detect on the glass-adhesive interface. It is important to note that only flaws at the interfaces can be detected. For the detection of flaws inside the adhesive layer, other methods are required, e.g. through thickness resonance method. For testing adhesively bonded joints in glass façades, the pulseecho ultrasonic method should be used in conjunction with matrix array transducers in order to cover larger areas and to produce easily understandable C-scan figures.

\section{REFERENCES}

[1] Roach, D., Rackow, K., Duvall, R. (2010). Innovative use of adhesive interface characteristics to nondestructively quantify 
the strength of bonded joints. 10th ECNDT Moscow Conference Proceedings.

[2] Budzik, M.K., Mascaro, B., Jumel, J., Castaings, M., Shanahan, M.E.R. (2012). Monitoring of crosslinking of a DGEBAPAMAM adhesive in composite/aluminium bonded joint using mechanical and ultra-sound techniques. International Journal of Adhesion and Adhesives, vol. 35, p. 120-128, DOl:10.1016/j.jjadhadh.2012.02.009.

[3] Shui, G., Wang, Y.S., Huang, P., Qu, J. (2015). Nonlinear ultrasonic evaluation of the fatigue damage of adhesive joints. NDT \& E International, vol. 70, p. 9-15, D0l:10.1016/j. ndteint.2014.11.002.

[4] Yan, D., Neild, S.A., Drinkwater, B.W. (2012). Modelling and measurement of the nonlinear behaviour of kissing bonds in adhesive joints. NDT \& E International, vol. 47, p. 18-25, DOI:10.1016/j.ndteint.2011.12.003.
[5] Titov, S.A., Maev, R.G., Bogachenkov, A.N. (2008). Pulse-echo NDT of adhesively bonded joints in automotive assemblies. Ultrasonics, vol. 48, no. 6-7, p. 537-546, D0l:10.1016/j. ultras.2008.07.001.

[6] Korzeniowski, M., Piwowarczyk, T., Maev, R.G. (2014). Application of ultrasonic method for quality evaluation of adhesive layers. Archives of Civil and Mechanical Engineering, vol. 14, no. 4, p. 661-670, D0l:10.1016/j.acme.2013.10.013.

[7] Kumar, R.L.V., Bhat, M.R., Murthy, C.R.L. (2013). Some studies on evaluation of degradation in composite adhesive joints using ultrasonic techniques. Ultrasonics, vol. 53, no. 6, p. 1150-1162, D0I:10.1016/j.ultras.2013.01.014.

[8] Castaings, M. (2014). SH ultrasonic guided waves for the evaluation of interfacial adhesion. Ultrasonics, vol. 54, no. 7 , p. 1760-1775, D0I:10.1016/j.ultras.2014.03.002. 